\title{
A METHOD OF TWO-LEVEL SIMPLIFICATION OF BOOLEAN FUNCTIONS
}

\author{
TOSHIO UMEZAWA
}

Dedicated to Professor K. Noshino on his sixtieth birthday

There are a number of methods to find minimal two-level forms for a given Boolean function, e g. Harvard's group [1], Veitch [2], Quine [3], [4], Karnaugh [5], Nelson [6], [7] etc.. This paper presents an approach which is suitable for mechanical or automatic computation, as the Harvard method and the Quine method are so. On the other hand, it shares the same property as the Veitch method in the sense that some of essential prime implicants may be found before all prime implicants are computed. It also adopts the procedure to reduce the necessary steps for computation which is shown in Lawler [8]. The method described is applicable to the interval of Boolean functions $f, g$ such that $f$ implies $g$ where for simplification of sum form the variables occurring in $g$ also occur in $f$ and for product form the variables in $f$ also occur in $g$.

\section{$\S 1$. Terminology and theorems}

A logical variable with or without - (negation) is called a primary. We make the conventions: $f, g$, and $h$ with or without suffixes are Boolean functions which are neither identically equal to 1 nor identically equal to 0 and $\varphi, x$ with or without suffixes are Boolean functions which have the just mentioned property and are constructed from primaries by a finite number of applications of + (multi-variable or), · (multi-variable and) and (if necessary) the auxiliary symbols $($,$) .$

We write $\varphi_{1}, \varphi_{2}, \ldots, \varphi_{n}$ in place of $\varphi_{1} \cdot \varphi_{2} \cdots \cdots \varphi_{n}$. For an $f$ which is primary, we define $f$ to be both a $\sigma$-form and a $\pi$-form. For any $f$ which is not primary, $f$ is defined to be a $\sigma$-form or $\pi$-form if the last application of logical operation is + or $\cdot$ respectively. We call $\varphi_{1}+\cdots+\varphi_{n}$ the sum of

Received June 11, 1966. 
$\varphi_{1}, \ldots, \varphi_{n}$ and $\varphi_{1}, \varphi_{2} \ldots \varphi_{n}$ the product of $\varphi_{1}, \varphi_{2}, \ldots, \varphi_{n}$.

We represent by $f \Rightarrow g$ that $f$ implies $g$ in the two-valued logic. $f \Rightarrow h \Rightarrow g$ represents that $f \Rightarrow h$ and $h \Longrightarrow g$. The number of literals occurring in $h$ is indicated by lit $(h)$. We say that $h$ is minimal in a set if lit $(h)$ is the least in the set of $\operatorname{lit}(f)$ 's where $f$ is any element of the set. $[f ; g]$ is defined to be the set of two-level $\varphi$ 's such that $f \Rightarrow \varphi \Rightarrow g$. The set of all variables occurring in $h$ is indicated by $V(h) . \sigma(f ; g)$ is defined to be the set of $\varphi$ 's which are minimal in the set of functions which are $\sigma$-forms and in $[f ; g]$. $\pi(f ; g)$ is similarly defined. $\varphi$ is called a prime implicant for $g$ in $[f ; g]$ if $\varphi \in[f ; g]$ and there is no $\varphi^{\prime}$ in $[f ; g]$ such that $\varphi \Rightarrow \psi^{\prime}$ and $\operatorname{lit}\left(\varphi^{\prime}\right)<\operatorname{lit}(\varphi)$. Similarly $\varphi$ is called a prime implicant for $f$ in $[f ; g]$ if $\varphi \in[f ; g]$ and there is no $\varphi^{\prime}$ in $[f ; g]$ such that $\varphi^{\prime} \Rightarrow \varphi$ and $\operatorname{lit}\left(\varphi^{\prime}\right)<\operatorname{lit}(\varphi)$.

Let the principal disjunctive normal form (p.d.n.f.) of $f$ be $m_{1}+\cdots+m_{k}$ and the principal conjunctive normal form (p.c.n.f.) of $g$ be $M_{1} M_{2} \cdots M_{l}$. Instead of $[f ; g], \sigma(f ; g), \pi(f ; g)$ we often write the expressions obtained from them substituting $\Phi$ for $f$ or $\Theta$ for $g$ where $\Phi$ is the set of suffixes of $m_{1}, \ldots, m_{k}$ and $\Theta$ the set of suffixes of $M_{1}, \ldots, M_{l}$. The first range of $\varphi$ with respect to $f$ or simply the first range of $\varphi$ is the set of $i$ 's such that $m_{i} \Longrightarrow \varphi$. Similarly the second range of $\varphi$ with respect to $g$ or simply the second range of $\varphi$ is the set of $i$ 's such that $\varphi \Rightarrow M_{i}$. [D; $\left.\Theta\right]$ where $\Phi$ and $\Theta$ are the first and second range of $\varphi$ respectively is called the range of $\varphi$ with respect to $(f, g)$ or simply the range of $\varphi$.

\subsection{If $\varphi \in \sigma(f ; g)$, then $V(\varphi) \subseteq V(g)$.}

Proof. Let $\varphi$ be an element of $\sigma(f ; g)$. There are $\varphi_{1}, \ldots, \varphi_{n}$ of $\pi$-forms such that $\varphi$ is $\varphi_{1}+\cdots+\varphi_{n}$. Since $\varphi$ is a two-level function, $\varphi_{1}, \ldots, \varphi_{n}$ are products of primaries. Let $\varphi_{i}^{\prime}$ be the function obtained from $\varphi_{i}$ by deleting all primaries whose variables are not in $V(g)$ and let $\varphi^{\prime}$ be $\varphi_{1}^{\prime}+\cdots+\varphi_{n}^{\prime}$. Since $\varphi_{i} \Longrightarrow g$, we obtain $\varphi_{i}^{\prime} \Rightarrow g$ by setting the values of primaries whose variables are not in $V(g)$ to be 1 . Hence it holds that $\varphi^{\prime} \Longrightarrow g$. Clearly $f \Longrightarrow \varphi^{\prime}$ and so $\varphi^{\prime} \in[f ; g]$. If $V(\varphi)-V(g)$ is not empty, then $\operatorname{lit}\left(\varphi^{\prime}\right)<\operatorname{lit}(\varphi)$ and hence contradicts to the definition of $\varphi$. Therefore $V(\varphi)-V(g)$ is empty.

1. 2. If $\varphi \in \pi(f ; g)$, then $V(\varphi) \subseteq V(f)$.

Proof. It is analogous as above. 
The next is essentially due to the lemma 2 in Lawler [8] $\S 4$.

1. 3. Let $[f ; g] \subseteq\left[f_{1} ; g_{1}\right]$. If the intersection of $\sigma\left(f_{1} ; g_{1}\right)$ and $[f ; g]$ is not empty, then $\sigma(f ; g)=\sigma\left(f_{1} ; g_{1}\right) \cap[f ; g]$ where $\cap$ denotes the intersection. Similarly, if the intersection of $\pi\left(f_{1} ; g_{1}\right)$ and $[f ; g]$ is not empty, then $\pi(f ; g)$ $=\pi\left(f_{1} ; g_{1}\right) \cap[f ; g]$.

1. 4. Let $V(g) \subseteq V(f)$ and the p.d.n.f. of $f$ be $m_{\mathrm{t}}+\cdots+m_{k}$. Further, let $\Gamma$ be the set of all prime implicants for $g$ in $\left[m_{r} ; g\right]$ where $m_{r}$ runs through $\left\{m_{1}, \ldots, m_{k}\right\}$. Then in order that $\varphi \in \sigma(f ; g)$, it is necessary and sufficient that $\varphi$ is minimal in the set of $\varphi_{1}+\cdots+\varphi_{n}$ in $[f ; g]$ where $\varphi_{1}, \ldots, \varphi_{n} \in \Gamma$.

Proof. Take any $\varphi$ in $\sigma(f ; g)$. There are $\varphi_{1}, \ldots, \varphi_{n}$ of $\pi$-forms such that $\varphi$ is $\varphi_{1}+\cdots+\varphi_{n}$. Since $f \Longrightarrow \varphi$, it holds for any $m_{j}(j=1, \ldots, k)$ that if $m_{j}=1$, then $\varphi=1$. By $1.1, V(\varphi) \subseteq V(g)$ and so, in virtue of the assumption, $V(\varphi) \subseteq V(f)$. Hence the values of all $\varphi_{i}^{\prime}$ 's $(i=1, \ldots, n)$ are determined. Since $\varphi_{1}+\cdots+\varphi_{n}=1$, there exists a $\varphi_{i}$ such that $\varphi_{i}=1$. Therefore for any $m_{j}$ there is a $\varphi_{i}$ such that $m_{j} \Rightarrow \varphi_{i}$. Now let $\bar{T}_{i}$ be the set of $m_{j}(1 \leq j \leq k)$ such that $m_{j} \Rightarrow \varphi_{i} . \quad \bar{\Psi}_{i}$ is not empty, otherwise $m_{1}+\cdots+m_{k} \Rightarrow \varphi_{1}+\cdots+$ $\varphi_{i-1}+\varphi_{i-1}+\cdots+\varphi_{n} \Rightarrow g$ and $\operatorname{lit}\left(\varphi_{1}+\cdots+\varphi_{i-1}+\varphi_{i+1}+\cdots+\varphi_{n}\right)<\operatorname{lit}(\varphi)$ and so it contradicts to the definition of $\varphi$. Hence for any $\varphi_{i}(1 \leq i \leq n)$, there is a $m_{j}$ such that $m_{j} \Rightarrow \varphi_{i}$. Furthermore, $\varphi_{i}$ is a prime implicant for $g$ in $\left[m_{j}: g\right]$, since otherwise there is a $\varphi_{i}^{\prime}$ such that $\varphi_{i}^{\prime} \in\left[\varphi_{i} ; g\right]$ and $\operatorname{lit}\left(\varphi_{i}^{\prime}\right)<\operatorname{lit}\left(\varphi_{i}\right)$ and hence $f \Rightarrow \varphi_{1}+\cdots+\varphi_{i-1}+\varphi_{i}^{\prime}+\varphi_{i+1}+\cdots+\varphi_{n} \Rightarrow g$ and lit $\left(\varphi_{1}+\cdots+\varphi_{i-1}+\right.$ $\left.\varphi_{i}^{\prime}+\varphi_{i+1}+\cdots+\varphi_{n}\right)<\operatorname{lit}(\varphi)$ which also contradicts to the definition of $\varphi$. Therefore $\varphi_{i} \in \Gamma(i=1, \ldots, n)$. Since $\varphi$ is minimal in the set of functions which are $\sigma$-forms and in $[f ; g], \varphi$ is also minimal in the set of the possible sums of elements of $\Gamma$ which is in $[f ; g]$. Conversely let $\varphi$ be minimal in the set of $\varphi_{1}+\cdots+\varphi_{n}$ in $[f ; g]$ where $\varphi_{1}, \ldots, \varphi_{n} \in \Gamma$. If $\varphi$ is not in $\sigma(f ; g)$, then there is a $\varphi^{\prime}$ such that $\varphi^{\prime} \in \sigma(f ; g)$ and $\operatorname{lit}\left(\varphi^{\prime}\right)<\operatorname{lit}(\varphi)$. From the above proven facts, $\varphi^{\prime}$ is a sum of elements of $\Gamma$ in $[f ; g]$ and hence $\operatorname{lit}(\varphi) \leq \operatorname{lit}\left(\varphi^{\prime}\right)$ by the definition of $\varphi$. Hence we have a contradiction. Consequently $\varphi \in \sigma(f ; g)$.

1.5. Let $V(f) \subseteq V(g)$ and the p.c.n.f. of $g$ be $M_{1} M_{2} \cdots M_{l}$. And let $\Gamma$ be the set of all prime implicants for $f$ in $\left[f ; M_{r}\right]$ where $M_{r}$ runs through $\left\{M_{1}, \ldots, M_{l}\right\}$. Then in order that $\varphi \in \pi(f ; g)$, it is necessary and sufficient that $\varphi$ is minimal in the set of $\varphi_{i} \varphi_{2} \ldots \varphi_{n}$ in $[f ; g]$ where $\varphi_{1}, \ldots, \varphi_{n} \in \Gamma$. 
Proof. It is similar as in 1.4 .

\section{§ 2. Algorithm}

For any $f, g$ which are neither identically 1 nor identically 0 and further the variables occurring in $g$ also occur in $f$, we give a procedure to find the minimal two-level $\varphi$ 's of $\sigma$-forms such that $f \Longrightarrow \varphi \Longrightarrow g$.

I 1. Compute the principal disjunctive normal form of $f, m_{1}+\cdots+m_{k}$, and the principal conjunctive normal form of $g, M_{1} M_{2} \cdots M_{l}$.

2. Seek all primaries occurring both in $m_{i}$ and in $M_{j}$ for all pairs of $(i, j)$ and their ranges. The table of primaries and the ranges obtained is called $\Pi$.

3. Let $\emptyset$ be $\{1,2, \ldots, k\}$ and $\Sigma$ be empty. Furthermore, let $\Gamma_{1}, \Gamma_{2}$ be empty tables. Take an $i$ in $\Phi$ arbitrarily.

4. Take the primaries whose first ranges contain $i$ and seek the set, $A$, of products such that their second ranges are $g$ and for any two of them the one does not imply the other.

5. If the first range of a product in $A$ is a subset of the first range of another in $\Lambda$ which has fewer literals, then delete the product from $A$.

6. From $\Phi$, delete all $j$ 's such that $m_{j} \Rightarrow \varphi$ where $\varphi$ is one of the minimals in $A$.

7. If $\Lambda$ consists of only one element, then transfer to the step II 1. Otherwise, transfer to the next.

8. Add the elements of $A$ to the table $\Gamma_{2}$ with their ranges except ones already written in $\Gamma_{2}$. Add the $i$ to $\Sigma$.

9. If $\Phi$ is not empty, then take an $i$ from $\Phi$ and return to I 4. Otherwise, transfer to the next.

10. If $\Gamma_{2}$ is not empty, then transfer to the next. Otherwise, the sum of products in $\Gamma_{1}$ is the minimal function required.

11. If there is an $i$ contained in the first range of only one element in $\Gamma_{2}$, then take the $i$ and return to I 5. Otherwise, transfer to the next.

12. Take the products both in $\Gamma_{2}$ and in $[\Sigma ; g]$ and seek minimals in the set of the sums of them which are in $[\Sigma ; g]$. If there is, among them, a minimal whose first range is the total of first ranges of all products in $\Gamma_{2}$, then make the sum of such a minimal and of all products in $\Gamma_{1}$. The functions thus obtained for all of such minimals are ones required. If there is no such. 
minimal, then take an $i$ which is not in $\Sigma$ and is contained in the first range of a product in $\Gamma_{2}$ and return to $I 5$.

II 1. From $\Sigma$ and the first ranges of any elements in $\Pi$ and $I_{2}^{*}$, delete all $j$ 's such that $m_{j} \Longrightarrow \varphi$ where $\varphi$ is the product, and further add the $\varphi$ to $\Gamma_{1}$.

2. If the first range of a product in $\Gamma_{2}$ is a subset of the first range of another in $\Gamma_{2}$ which has fewer literals, then delete the product. Return to I 9.

Dually we have the procedure for forms of products of sums by the following modification where the variables occurring in $f$ also occur in $g$.

The words "first, product, sum" are replaced by "second, sum, product" respectively. Also " $m_{j} \Rightarrow \varphi$ " is replaced by " $\varphi \Longrightarrow M_{j}$ ". Furthermore, in the step I $4, g$ is replaced by $f$.

Explanation. We restrict ourselves to the case of $\sigma$-forms. Let the p.d.n.f. of $f$ be $m_{1}+\cdots+m_{k}$. Let $\Sigma$ be a subset of $\left\{m_{1}, \ldots, m_{k}\right\}$. If there is an elements of $\sigma(\Sigma ; g)$ which is also in $[f ; g]$, then, by $1.3, \sigma(f ; g)$ is equal to all of such elements of $\sigma(\Sigma ; g)$. Hence, in order to reduce the labour of computation, it is necessary to find a suitable subset of $\left\{m_{1}, \ldots, m_{k}\right\}$. Here $\Sigma$ is given as the set of $m_{i}$ 's taken from the residue of $\left\{m_{1}, \ldots, m_{k}\right\}$ obtained by deleting $m_{j}$ such that $m_{j} \Rightarrow \varphi$ where $\varphi$ is minimal in the set of prime implicants for $g$ in $\left[m_{r} ; g\right]$ for a $m_{r}$ in $\Sigma$ already obtained.

The prime implicants for $g$ in $\left[m_{r} ; g\right]$ are computed by the method described in the step I 4. If the set $\Lambda$ is an unit set, then the prime implicant is essential. In combining prime implicants so as to get minimal functions, it is not necessary to consider $m_{i}$ 's which imply an essential prime implicant. Hence we eliminate such $m_{i}$ 's from $\Sigma, \Pi$. $\Gamma_{1}$ is intended for the table of essential prime implicants and $\Gamma_{2}$ the table of non-essential prime implicants. These are given in the step II 1.

The table $\Gamma_{2}$ thus obtained contains all prime implicants for $g$ in $\left[m_{r} ; g\right]$ where $m_{r}$ is any element of $\Sigma$. If $\Gamma_{2}$ is empty, then the sum, $\varphi$, of products in $\Gamma_{1}$ has the property that $m_{1}+\cdots+m_{k} \Rightarrow \varphi$. Hence $\varphi$ is the minimal required. Let $\Gamma_{2}$ be not empty. It is not certain whether these prime implicants are all non-essential or not. Hence, in the step I 11, we take possible essential prime implicants and distinguish essential prime implicants from non-essential ones. Then we seek minimals in the set of sums in $[\Sigma ; g]$ of non-essential prime implicants in $[\Sigma ; g]$. If there is a minimal in $[\Xi ; g]$ where $\Xi$ is the set of 
$j$ 's $(1 \leq j \leq k)$ such that $m_{j}$ does not imply any element in $\Gamma_{1}$, then the sum of such a minimal and of all products in $\Gamma_{1}$ is in $[f ; g] . \Xi$ is given as the total of first ranges of all elements in $\Gamma_{2}$. Then all of such sums are the minimal functions required. If there is no such minimal, then we enlarge $\Sigma$ and repeat these steps. The step I 12 reflects these circumstances.

\section{§ 3. Examples}

The first example is the simplification of $a b e+\bar{a} d e+\bar{b} c \bar{e}+\bar{a} \bar{c} \bar{d} \vec{e}+a \bar{d} \bar{c} \bar{d}+a b c d$, which was also used as examples of the Quine method, the Harvard method and the Veitch method in p. 73, p. 79 and p. 92 in [9] respectively. We seek the minimal equivalents of $\sigma$-forms by our method.

Let $f$ and $g$ be the given function. The p.d.n.f. of $f$ is $m_{1}+m_{2}+\cdots+m_{17}$ where $m_{1}$ is $\bar{a} \bar{b} \bar{c} \bar{d} \bar{e}, m_{2} \bar{a} \bar{b} \bar{c} d e, m_{3} \bar{a} \bar{b} c \bar{d} \bar{e}, m_{4} \bar{a} \bar{b} c d \bar{e}, m_{5} \bar{a} \bar{b} c d e, m_{6} \bar{a} b \bar{c} \bar{d} \bar{e}, m_{7} \bar{a} b \bar{c} d e$, $m_{8} \bar{a} b c d e, m_{9} a \bar{b} \bar{c} \bar{d} \bar{e}, m_{10} a \bar{b} \bar{c} \bar{d} e, m_{11} a \bar{b} c \bar{d} \bar{e}, m_{12} a \bar{b} c d \bar{e}, m_{13} a b \bar{c} \bar{d} e, m_{14} a b \bar{c} d e, m_{15}$ $a b c \bar{d} e, m_{16} a b c d \bar{e}, m_{1 i} a b c d e$. The p.c.n.f. of $g$ is $M_{1} M_{2} \cdots M_{15}$ where $M_{1}$ is $a+b+c+d+\bar{e}, M_{2} a+b+c+\bar{d}+e, M_{3} a+b+\bar{c}+d+\bar{e}, M_{1} a+\bar{b}+c+d+\bar{e}$, $M_{5} a+\bar{b}+c+\bar{d}+e, M_{6} a+\bar{b}+\bar{c}+d+e, M_{7} a+\bar{b}+\bar{c}+d+\bar{e}, M_{8} a+\bar{b}+\bar{c}+\bar{d}$ $+e, M_{9} \bar{a}+b+c+\bar{d}+e, \quad M_{10} \bar{a}+b+c+\bar{d}+\bar{e}, \quad M_{11} \bar{a}+b+\bar{c}+d+\bar{e}, M_{12} \bar{a}+b$

$+\bar{e}+\bar{d}+\bar{e}, M_{13} \bar{a}+\bar{b}+c+d+e, M_{14} \bar{a}+\bar{b}+c+\bar{d}+e, M_{15} \bar{a}+\bar{b}+\bar{c}+d+e$.

Then the table $I$ is given as follows.

II: primaries ranges

$\begin{array}{ll}a & {[9,10,11,12,13,14,15,16,17 ; 1,2,3,4,5,6,7,8]} \\ \bar{a} & {[1,2,3,4,5,6,7,8 ; 9,10,11,12,13,14,15]} \\ b & {[6,7,8,13,14,15,16,17 ; 1,2,3,9,10,11,12]} \\ \bar{b} & {[1,2,3,4,5,9,10,11,12 ; 4,5,6,7,8,13,14,15]} \\ c & {[3,4,5,8,11,12,15,16,17 ; 1,2,4,5,9,10,13,14]} \\ \bar{c} & {[1,2,6,7,9,10,13,14 ; 3,6,7,8,11,12,15]} \\ d & {[2,4,5,7,8,12,14,16,17 ; 1,3,4,6,7,11,13,15]} \\ \bar{d} & {[1,3,6,9,10,11,13,15 ; 2,5,8,9,10,12,14]} \\ e & {[2,5,7,8,10,13,14,15,17 ; 2,5,6,8,9,13,14,15]} \\ \bar{e} & {[1,3,4,6,9,11,12,16 ; 1,3,4,7,10,11,12] .}\end{array}$

Let $\emptyset$ be $\{1,2,3, \ldots, 17\}$ and let $\Sigma, \Gamma_{1}, \Gamma_{2}$ be empty. First we take 1 from $\mathscr{D}$. Then the primaries whose first ranges contain 1 and their ranges are: 


$\begin{array}{ll}\bar{a} & {[1,2,3,4,5,6,7,8 ; 9,10,11,12,13,14,15]} \\ \bar{b} & {[1,2,3,4,5,9,10,11,12 ; 4,5,6,7,8,13,14,15]} \\ \bar{c} & {[1,2,6,7,9,10,13,14 ; 3,6,7,8,11,12,15]} \\ \bar{d} & {[1,3,6,9,10,11,13,15 ; 2,5,8,9,10,12,14]} \\ \bar{e} & {[1,3,4,6,9,11,12,16 ; 1,3,4,7,10,11,12] .}\end{array}$

The prime implicants for $g$ in $[1 ; g]$ have $g$ as their second ranges and hence the primaries $\bar{d}$ and $\bar{e}$ essentially occur in them, since 2 and 1 in the second ranges of $\bar{d}$ and $\bar{e}$ respectively occur only once in the second ranges of the above shown primaries. Then they are $\bar{a} \bar{d} \bar{e}$ and $\bar{a} \bar{c} \bar{d} \bar{e}$ whose ranges are $[1,3$, $9,11 ; g]$ and $[1,6 ; g]$ respectively. We delete $1,3,9,16$ from $\Phi$ according to $\mathrm{I} 6$ and write $\bar{a} \bar{d} \bar{e}$ and $\bar{a} \bar{c} \bar{d} \bar{e}$ into the table $\Gamma_{2}$ and add 1 to $\Sigma$ by I 8. Then we take 2 from $\Phi$ and repeat these computations. The primaries which contain 2 in their first ranges are:

$$
\begin{array}{ll}
\vec{a} & {[1,2,3,4,5,6,7,8 ; 9,10,11,12,13,14,15]} \\
\bar{b} & {[1,2,3,4,5,9,10,11,12 ; 4,5,6,7,8,13,14,15]} \\
\vec{c} & {[1,2,6,7,9,10,13,14 ; 3,6,7,8,11,12,15]} \\
d & {[2,4,5,7,8,12,14,16,17 ; 1,3,4,6,7,11,13,15]} \\
e & {[2,5,7,8,10,13,15,17 ; 2,5,6,8,9,13,14,15] .}
\end{array}
$$

Among them, $\bar{a}, d$ and $e$ essentially occur in prime implicants for $g$ in $[2 ; g]$ because of 10,1 , and 2 in their second ranges. Their product $\bar{a} d e$ has $g$ as the second range and hence it is the unique prime implicant for $g$ in $[2 ; g]$. So $\bar{a} d e$ is an essential prime implicant for $g$ in $[f ; g]$. By the steps I 6 and II 1 , we delete $2,5,7,8$ from $\emptyset, \Sigma$, and the first ranges of any elements in $I$ and $I_{2}$. Add $\bar{a} d e$ to $\Gamma_{1}$. Next we take 4 from $\Phi$ and computing similarly, obtain $\bar{b} c \bar{e}$ and $\bar{a} \bar{b} c d$ as prime implicants for $g$ in $[4 ; g]$. Their ranges are $[3,4,11,12 ; g]$ and $[4 ; g]$ and hence, by $I 5, \bar{a} \bar{b} c d$ is eliminated. Therefore, by II 1 , we delete $3,4,11,12$ from the first ranges of elements in $\Pi$ and $\Gamma_{2}$ and add $\bar{a} c \bar{e}$ to $\Gamma_{1}$. From $\Phi, 3,4,11,12$ are deleted. Then the remaing $\Phi$ is the set of $6,10,13,14,15,16,17$. We take 6 and obtain $\bar{a} \bar{c} \bar{d} \bar{e}$ as the unique prime implicant for $g$ in $[6 ; g]$. Then 1,6 , the first range of $\bar{a} \vec{c} \vec{d} \vec{e}$, is eliminated from the first ranges of elements in $\Pi$ and $\Gamma_{2}$ and from $\Phi$ and $\Sigma . \quad \bar{a} \bar{c} \bar{d} \bar{e}$ with the empty first range is eliminated from $\Gamma_{2}$. Computing in this way, we may obtain the following. 
$\Sigma: 10,14,16$.

$\Gamma_{1}: \bar{a} d e, \bar{b} c \dot{\bar{e}}, \bar{a} \bar{c} \bar{d} \bar{e}$.

$\Gamma_{2}: \bar{b} \bar{d} \bar{e}[9 ; g], a \bar{b} \bar{c} \bar{d}[9,10 ; g], a \bar{c} \bar{d} e[10,13 ; g], a b e[13,14,15,17 ; g]$, $b d e[14,17 ; g]$, abcd $[19,17 ; g]$, acde $[16 ; g]$.

Now $\Phi$ is empty. Since $\Gamma_{2}$ is not empty, we proceed to the step I 11.15 occurs only in the first range of abe. So regarding 15, we perform the same computations and obtain $a b e$ as the unique prime implicant for $g$ in $[15 ; g]$. The range is $[13,14,15,17 ; g]$. Hence we delete $13,14,15,17$ from $\Sigma$ and the first ranges of any elements in $\Pi$ and $\Gamma_{2}$. abe and bde are also eliminated from $\Gamma_{2}$. The elements both in $\Gamma_{2}$ and in $[10,16 ; g]$ are $a \bar{b} \bar{c} \bar{d}, a \bar{c} \bar{d} e, a b c d$, and $a c d \bar{e}$. Then $a \bar{b} \bar{c} \bar{d}+a b c d$ is minimal in the set of the sums of them which are in $[10,16 ; g]$ and the first range of it is $\{9,10,16\}$, the total of first ranges of all products in $\Gamma_{2}$. Such minimals are $a \bar{b} \bar{c} \bar{d}+a b c d, a \bar{b} \bar{c} \bar{d}+a c d \bar{e}$. Hence, the minimal functions required are the sum of them and of elements in $\Gamma_{1}$, i.e. $\bar{a} d e+\bar{b} c \bar{e}+\bar{a} \bar{c} \bar{d} \bar{e}+a \bar{b} \bar{c} \bar{d}+a b c d$ and $\bar{a} d e+\bar{b} c \bar{e}+\bar{a} \bar{c} \bar{d} \bar{e}+a \bar{b} \bar{c} \bar{d}+a c d \bar{e}$.

Next we give an example of simplification of $\pi$-form under the condition that $f$ and $g$ are not equal. The given function is $A x+B y+C z$ where $x y, x z$. and $y z$ are redundant combinations (don't cares). This was used as an example of the Veitch method in [9]. Then we take as $f(A x+B y+C z) \overline{(x y+x z+y z)}$ and as $g A x+B y+C z+x y+x z+y z$.

The p.d.n.f. of $f$ is $m_{1}+m_{2}+\cdots+m_{12}$ where $m_{1}$ is $\bar{A} \bar{B} C \bar{x} \bar{y} z, m_{2} \bar{A} B \bar{C} \bar{x} y \bar{z}$, $m_{3} \bar{A} B C \bar{x} \bar{y} z, m_{4} \bar{A} B C \bar{x} y \bar{z}, m_{5} A \bar{B} \bar{C} x \bar{y} \bar{z}, m_{6} A \bar{B} C \bar{x} \bar{y} z, m_{7} A \bar{B} C x \bar{y} \bar{z}, m_{3} A B \bar{C} \bar{x} y \bar{z}$, $m_{9} A B \bar{C} x \bar{y} \bar{z}, m_{10} A B C \bar{x} \bar{y} z, m_{11} A B C \bar{x} y \bar{z}, m_{12} A B C x \bar{y} \bar{z}$ and the p.c.n.f. of $g$ is $M_{1} M_{2} \cdots M_{20}$ where $M_{1}$ is $A+B+C+x+y+z, M_{2} A+B+C+x+y+\bar{z}, M_{3}$ $A+B+C+x+\bar{y}+z, \quad M_{4} \quad A+B+C+\bar{x}+y+z, \quad M_{5} \quad A+B+\bar{C}+x+y+z, \quad M_{6}$ $A+B+\bar{C}+x+\bar{y}+z, \quad M_{7} A+B+\bar{C}+\bar{x}+y+z, \quad M_{8} A+\bar{B}+C+x+y+z, \quad M_{9}$ $A+\bar{B}+C+x+y+\bar{z}, M_{10} A+\bar{B}+C+\bar{x}+y+z, M_{11} A+\bar{B}+\bar{C}+x+y+z, M_{12}$ $A+\bar{B}+\bar{C}+\bar{x}+y+z, M_{13} \bar{A}+B+C+x+y+z, M_{14} \bar{A}+B+C+x+y+\bar{z}, \quad M_{15}$ $\bar{A}+B+C+x+\bar{y}+z, \quad M_{16} \bar{A}+B+\bar{C}+x+y+z, \quad M_{17} \bar{A}+B+\bar{C}+x+\bar{y}+z, M_{18}$ $\bar{A}+\bar{B}+C+x+y+z, M_{19} \bar{A}+\bar{B}+C+x+y+\bar{z}, M_{20} \bar{A}+\bar{B}+\bar{C}+x+y+z$.

The table $\Pi$ is given as follows.

$\pi$ : 


$\begin{array}{ll}A & {[5,6,7,8,9,10,11,12 ; 1,2,3,4,5,6,7,8,9,10,11,12]} \\ \bar{A} & {[1,2,3,4 ; 13,14,15,16,17,18,19,20]} \\ B & {[2,3,4,8,9,10,11,12 ; 1,2,3,4,5,6,7,13,14,15,16,17]} \\ \bar{B} & {[1,5,6,7 ; 8,9,10,11,12,18,19,20]} \\ C & {[1,3,4,6,7,10,11,12 ; 1,2,3,4,8,9,10,13,14,15,18,19]} \\ \bar{C} & {[2,5,8,9 ; 5,6,7,11,12,16,17,20]} \\ x & {[5,7,9,12 ; 1,2,3,5,6,8,9,11,13,14,15,16,17,18,19,20]} \\ \bar{x} & {[1,2,3,4,6,8,10,11 ; 4,7,10,12]} \\ y & {[2,4,8,11 ; 1,2,4,5,7,8,9,10,11,12,13,14,16,18,19,20]} \\ \bar{y} & {[1,3,5,6,7,9,10,12 ; 3,6,15,16]} \\ z & {[1,3,6,10 ; 1,3,4,5,6,7,8,10,11,12,13,15,16,17,18,20]} \\ \bar{z} & {[2,4,5,7,8,9,11,12 ; 2,9,14,19] .}\end{array}$

Let $\Phi$ be $\{1,2, \ldots, 20\}$ and $\Sigma, \Gamma_{1}, \Gamma_{2}$ be empty. First we take 1 from $\emptyset$. Then the primaries whose second ranges contain 1 and their ranges are:

$\begin{array}{ll}A & {[5,6,7,8,9,10,11,12 ; 1,2,3,4,5,6,7,8,9,10,11,12]} \\ B & {[2,3,4,8,9,10,11,12 ; 1,2,3,4,5,6,7,13,14,15,16,17]} \\ C & {[1,3,4,6,7,10,11,12 ; 1,2,3,4,8,9,10,13,14,15,18,19]} \\ x & {[5,7,9,12 ; 1,2,3,5,6,8,9,11,13,14,15,16,17,18,19,20]} \\ y & {[2,4,8,11 ; 1,2,4,5,7,8,9,10,11,12,13,14,16,18,19,20]} \\ z & {[1,3,6,10 ; 1,3,4,5,6,7,8,10,11,12,13,15,16,17,18,20]}\end{array}$

The prime implicants for $f$ in $[f ; 1]$ and their ranges are $A+B+C[f: 1$, $2,3,4], B+C+x[f ; 1,2,3,13,14,15], A+C+y[f ; 1,2,4,8,9,10], C+x+y$ $[f ; 1,2,8,9,13,14,18,19], A+B+z[f ; 1,3,4,5,6,7], B+x+z[f ; 1,3$, $5,6,13,15,16,17], A+y+z[f ; 1,4,5,7,8,10,11,12], x+y+z[f ; 1,5,8$, $11,13,16,18,20]$. $\Phi$ becomes empty by deleting the second ranges of them and hence they are all of elements in $\Gamma_{2}, 1$ is added to $\Sigma$.

$12,17,19,20$ occur once respectively in the second ranges of elements in $\Gamma_{2}$. We obtain $A+\bar{x}$ and $A+y+z$ from the computation regarding $12, B+\bar{y}$ and $B+x+z$ regarding $17, C+\bar{z}$ and $C+x+y$ regarding 19. Among them, $A+\bar{x}, B+\bar{y}$, and $C+\bar{z}$ are added to $\Gamma_{2}$ where their ranges are $[f ; 4,7,10,12]$, $[f ; 3,6,15,17]$, and $[f ; 2,9,14,19]$ respectively. Regarding 20 , we obtain $x+y+z$ with the range $[f ; 1,5,8,11,13,16,18,20]$. From $\Sigma$ which is $\{1$, $12,17,19\}$ and from the second ranges of any elements in $\Pi$ and $\Gamma_{2}$, we delete 
$1,5,8,11,13,16,18,20$ and $x+y+z$ is added to $\Gamma_{1} . \quad x+y+z$ in $\Gamma_{2}$ is eliminated because of the empty second range. In virtue of II $2, C+x+y, B+x+z$, and $A+y+z$ are deleted from $\Gamma_{2}$. Then $\Sigma$ is $\{12,17,19\}$ and $\Gamma_{2}$ newly obtained is $A+B+C[f ; 2,3,4], B+C+x[f ; 2,3,14,15], A+C+y[f ; 2,4,9,10]$, $A+B+z[f ; 3,4,6,7], A+\bar{x}[f ; 4,7,10,12], B+\bar{y}[f ; 3,6,15,17], C+\bar{z}$ $[f ; 2,9,14,19]$. Again we apply I 11 and find that $12,17,19$ occur once respectively. Regarding 12 , we obtain quite similarly $A+\vec{x}$ and $A+y+z$ except that their ranges are the same. Hence, in this case, $A+y+z$ is deleted by $\mathrm{I} 5$ and $A+\bar{x}$ is added to $\Gamma_{1} . \quad 12$ in $\Sigma$ and $A+\bar{x} A+y+z$ in $\Gamma_{2}$ are deleted by II 1 . Computing in the same way, we obtain $B+\bar{y}$ and $C+\bar{z}$ as elements in $I_{1}$. Then $\Sigma$ and $\Gamma_{2}$ become empty. Hence, by I 10, the minimal function required is $(x+y+z)(A+\bar{x})(B+\bar{y})(C+\bar{z})$.

\section{REFERENCES}

[1] Staff of Harvard Computation Laboratory, Synthesis of electronic computing and control circuits, Harvard University Press, Cambridge, 1951.

[2] E. W. Veitch, A chart method for simplifying truth functions, Proc. Association for Computing Machinery Conference, May 2-3, 1952, pp. 127-133.

[3] W. V. Quine, The problem of simplifying truth-functions, Amer. Math. Monthly, 59 (1952), pp. 521-531.

[4] W. V. Quine, A way to simplify truth functions, Amer. Math. Monthly, 62 (1955), pp. $627-631$.

[5] M. Karnaugh, The map method for synthesis of combinational logic circuits, Trans, A mer. Inst. Elec. Engrs., 72(I) (1953), pp. 593-599.

[6] R. J. Nelson, Simplest normal truth functions, J. Symbolic Logic, 20 (1955), pp. 105-108.

[7] R. J. Nelson, Weak simplest normal truth functions, J. Symbolic Logic, 20 (1955), pp. 232-234.

[8] E. L. Lawler, An approach to multilevel Boolean minimization, J. Assoc. for Computing Machinery, 11 (1964), pp. 283-295.

[9] M. Phister, Logical design of digital computers, John Wiley, New York, 1960.

\section{Shizuoka University}

